\title{
Preparation and Characterization of a Novel Absorber for Formaldehyde
}

\author{
Tianhui HUANG \\ China Tobacco Guangxi Industrial Co.LTD, South Beihu \\ Guangxi, China \\ e-mail:18677191775@163.com
}

\section{Yuan XI}

Key Laboratory for Biomechanics and Mechanobiology of Ministry of Education

School of Biological Science and Medical Engineering, Beihang University, No.37 Xueyuan

Beijing, China

e-mail: 1152363466@qq.com

\author{
Hong LIU \\ China Tobacco Guangxi Industrial Co.LTD., South Beihu, \\ Guangxi, China \\ e-mail:634087105@qq.com
}

\section{Yan LIU}

Key Laboratory for Biomechanics and Mechanobiology of Ministry of Education

School of Biological Science and Medical Engineering, Beihang University, No.37 Xueyuan

Beijing, China

e-mail: liuyan@buaa.edu.cn

\section{Gang ZHOU}

Key Laboratory for Biomechanics and Mechanobiology of Ministry of Education School of Biological Science and Medical Engineering, Beihang University, No.37 Xueyuan

Beijing, China

e-mail: zhougang@buaa.edu.cn

\begin{abstract}
Formaldehyde is one of the substances causing sick for human being.Small doses of formaldehyde can cause a variety of symptoms of physical discomfort. High dose and long time exposure to formaldehyde will increase the probability of cancer. However, the content of formaldehyde in the newly renovated room or near the new furniture is very high. To solve the formaldehyde, this paper developed a new kind of sodium carboxymethyl cellulose/carboxyl graphite oxide (CMC/GO-COOH) scaffold via a lyophilization method. Then, this synthetic scaffold was characterized by Fourier transform infrared spectroscopy (FT-IR), scanning electron microscope (SEM), hydrophilic test, mechanical property and in vitro detoxification test. There were covalent bonding and hydrogen bonding in the scaffold, indicating the strong interactions between $\mathrm{CMC}$ and GO-COOH. There were interconnected network in the structure of synthesized scaffold. The mechanical test suggested that the $\mathrm{CMC} / \mathrm{GO}-\mathrm{COOH}$ scaffold had excellent mechanical strength, which was $3.41 \pm 0.12 \mathrm{MPa}$ with shrink of $25 \%$. Furthermore, the adsorption of $\mathrm{CMC/GO-COOH}$ to formaldehyde wereup to $10.97 \pm 1.84 \mathrm{mg} / \mathrm{g}$. The resulting CMC/GO-COOH scaffold held great potential for adsorbing formaldehyde.
\end{abstract}

\section{Keywords-CMC/GO-COOH; scaffold ; formaldehyde}

\section{INTRODUCTION}

Formaldehyde is one of the substances causing sick for human being. People may feel uncomfortable when formaldehyde reaches a certain concentration in the room. If the formaldehyde concentration is greater than 0.08 milligram per stere, it can cause redness, itching, throat discomfort, hoarseness, sneezing, chest tightness, asthma or dermatitis. Newly renovated room contains higher level of formaldehyde, which is the main cause of many diseases. Long term exposure to formaldehyde increases the risk of Hodgkin's lymphoma, multiple myeloma, myeloid leukemia and other special cancer.

The adsorption formaldehyde has been investigated byseveral groups for a long time. Specifically, the porous detoxifying scaffold $[1,2]$ based on high specific surface area, had been proved to be an effective material in artificial kidneys. Activated carbon kept the first choice in environment detoxification. Nevertheless, the detoxification efficiency of activated carbon to formaldehyde was rather low. Graphite Oxide (GO)[3] attracted great attentions due to its unique thermal, mechanical and electrical properties. All these specific characteristics were attributed to its unique structure and oxygen-containing groups, such as epoxy, hydroxyl and carboxyl groups and so on. Carboxyl Graphite Oxide $(\mathrm{GO}-\mathrm{COOH})$ was important due to its high reactivity, dispersibility and stability. The GO-COOH showed better reactivity and stability than $\mathrm{GO}$ for further application.

Herein, $\mathrm{CMC}$ and $\mathrm{GO}-\mathrm{COOH}$ were combined to overcome their own disadvantages alone. GO was prepared by the modified Hummers method. CMC/GO-COOH scaffolds were prepared via a lyophilization method. The CMC/GO-COOH scaffold synthesized was characterized by FT-IR, SEM, mechanical stress. In vitro experiment, the adsorption of $\mathrm{CMC} / \mathrm{GO}-\mathrm{COOH}$ scaffolds for formaldehyde.

\section{MATERIALS AND METHODS}

Natural graphite powder (180 mesh) was obtained from Qingdao Black Dragon Graphite Co. Ltd., China. Chitosan, 
urea, creatinine, Vitamin B12 and $\beta 2-\mathrm{m}$ were all purchased from Sigma-Aldrich Inc., USA. Potassium permanganate (KMnO4), sulfuric acid (H2SO4, 98wt.\%), sodium nitrate (NaNO3), sodium hydroxide $(\mathrm{NaOH})$ and hydrogen peroxide (H2O2) were supplied by Aladdin Reagent Co. Ltd., China. All the chemicals were analytical grade and doubly deionized water was used throughout.

\section{A. Synthesis of Carboxyl Graphite Oxide}

GO was synthesized using the modified Hummers method. Natural graphite powder (2g), NaNO3 (1g) and $\mathrm{H} 2 \mathrm{SO} 4$ (98wt.\%, 46mL) were mixed in a round bottom flask, under magnetic stirring for $12 \mathrm{~h}$ at $-5^{\circ} \mathrm{C}$. Next, $\mathrm{KMnO} 4(6 \mathrm{~g})$ was added to the mixture gradually, and then the mixture was heated to $35^{\circ} \mathrm{C}$. After continuous stirring for $1 \mathrm{~h}$, distilled water $(46 \mathrm{~mL})$ was poured into the solution at $80^{\circ} \mathrm{C}$. After $30 \mathrm{~min}$, water $(280 \mathrm{~mL})$ along with $\mathrm{H} 2 \mathrm{O} 2(30 \mathrm{wt} . \%$, 40ml) was added under stirring. The reason adding $\mathrm{H} 2 \mathrm{O} 2$ was to remove the remaining of $\mathrm{KMnO} 4$. Subsequently, the mixture was filtered, and the obtained solid material was put into distilled water. Centrifugation was carried out for $30 \mathrm{~min}$ at 4000rpm and material obtained from centrifugation was put into water again. The procedure above was repeated until the $\mathrm{PH}$ of the material was neutralized up to 7 . Finally, the resultant product was dried at $50^{\circ} \mathrm{C}$ and $\mathrm{GO}$ was obtained in the form of dark brown slice.

The carboxyl of GO was activated by chloroacetic acids according to the reported method. The prepared GO (200mg) was suspended in distilled water $(100 \mathrm{~mL})$, followed by sonication for about $2 \mathrm{~h}$. Then, $\mathrm{NaOH}(12 \mathrm{~g})$ was added to the mixture under stirring. About 30min later, chloroacetic acids $(20 \mathrm{~g})$ was introduced into the mixture under strong basic condition. After continuous sonication for $2 \mathrm{~h}$, the resultant was purified by dialysis, washed with ethanol, and separated by centrifugation. Finally, the product was dried at $60^{\circ} \mathrm{C}$ and $\mathrm{GO}-\mathrm{COOH}$ was obtained.

\section{B. Synthesis of CMC/GO-COOH Scaffolds Carboxyl Graphite Oxide}

$\mathrm{CMC} / \mathrm{GO}-\mathrm{COOH}$ scaffolds were prepared via the double-lyophilization method, as shown in Fig.1. The process included two important steps, namely, the synthesis of $\mathrm{CS} / \mathrm{GO}-\mathrm{COOH}$ hydrogels, and subsequent doublelyophilization technique.

$\mathrm{CMC}(3.1 \mathrm{~g})$ was dissolved in distilled water $(5 \%, 55 \mathrm{~mL})$ solution under continuous stirring overnight. Meanwhile, a $1.0 \mathrm{mg} / \mathrm{mL}$ GO-COOH was added into the distilled water and followed by sonication for $2 \mathrm{~h}$. The ratio of $\mathrm{GO}-\mathrm{COOH}$ introduced into the mixture mainly depended on its dissolving capacity in water. In the pre-experiment, the solubility of $\mathrm{GO}-\mathrm{COOH}$ was $7.0 \mathrm{mg} / \mathrm{mL}$. Then, the CMC solution and $\mathrm{GO}-\mathrm{COOH}$ aqueous dispersion were mixed together under constant stirring. The mixture was agitated for $24 \mathrm{~h}$. Then, the mixed solution $(25 \mathrm{~mL})$ was transferred to a glass bottle $(20 \times 40 \mathrm{~mm})$, and frozen at minus $80^{\circ} \mathrm{C}$ for $10 \mathrm{~h}$. Subsequently, the sample was first lyophilized at minus $50^{\circ} \mathrm{C}$ condition. Finally, the CMC/GO-COOH scaffold was obtained.

\section{Characterization}

Fourier transform infrared spectrometer (FT-IR, Nicolet iS10, USA) was performed to analysis the interactions between $\mathrm{CMC}$ and $\mathrm{GO}-\mathrm{COOH}$. The FT-IR was tested in the range of $4000-500 \mathrm{~cm}-1$, at $1.5 \mathrm{~cm}-1$ resolution and averaging 100 scans.

The surface morphology of CMC/GO-COOH scaffold was observed with a Scanning Electron Microscope (SEM, FEI250, USA), with an accelerated voltage of 10KV. The average diameter of structure was measured from SEM images by Image Program.

The Dynamic Mechanical Analyzer (Electro-Force 3550, USA) was used to measure the dynamic stiffness of scaffolds synthesized. Scaffolds were loaded with the dynamic force until it was at strain of $90 \%$. More specifically, a starting force of $0 \mathrm{~N}$ was applied and then continuously increased with the movement of $0.5 \mathrm{~mm} / \mathrm{s}$ at a constant frequency of $1 \mathrm{HZ}$.

The Adsorption Experimentwas carried out by OSHA's titerimetricanalysis method[4] for formaldehyde.

\section{Statistical Analysis}

Data were pooled from at least three independent experiments and presented as mean \pm standard deviation $( \pm \mathrm{s})$ unless indicated otherwise. Differences between groups were analyzed using one-way analysis of variance. All the statistical analyses were performed with SPSS13.0. ${ }^{*} \mathrm{p}<0.05$ was considered statistically significant.

\section{RESUlts AND DisCUSSION}

It can be found the characteristic peak of alkoxy (C-O) was displayed at $1054 \mathrm{~cm}-1$ (shown in Fig1b). The absorption bands at $1736 \mathrm{~cm}-1$ and $1634 \mathrm{~cm}-1$ were attributed to the stretching vibrations of carbonyl and carboxylic groups, respectively. In Fig.1a, the absorbance band of CMC shifted. This result was attributed to the der Waals interactions between $\mathrm{GO}-\mathrm{COOH}$ and $\mathrm{CMC}$.The mechanical test suggested that the $\mathrm{CMC} / \mathrm{GO}-\mathrm{COOH}$ scaffold had excellent mechanical strength, which was $3.41 \pm 0.12 \mathrm{MPa}$ with shrink of $25 \%$. This could be ascribed to the strong interfacial interactions between $\mathrm{GO}-\mathrm{COOH}$ and the $\mathrm{CMC}$ matrix. In addition to the electrostatic interactions, there were the strong hydrogen bonding and covalent bonding formed between the carboxyl groups of $\mathrm{GO}-\mathrm{COOH}$ and hydroxyl groups of CMC matrix. Strong interfacial adhesion was established between both components. The pores in the scaffolds formed during the lyphilization process. And the average diameter corresponding to these scaffolds was $232 \pm 15 \mathrm{um}$. The adsorption of $\mathrm{CMC} / \mathrm{GO}-\mathrm{COOH}$ to formaldehyde wereup to $10.97 \pm 1.84 \mathrm{mg} / \mathrm{g}$. After adsorption experiment, there are many blocked, sealed tubular structures. This means that $\mathrm{CMC} / \mathrm{GO}-\mathrm{COOH}$ scaffold held great potential for adsorbing formaldehyde. 


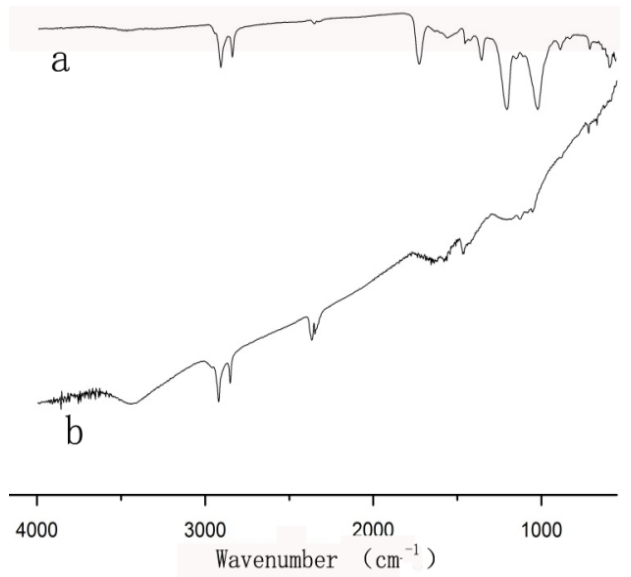

Figure 1. FT-IR curves of synthesized CMC/GO-COOHandGO-COOH scaffold were shown in Fig. 3(a-d), respectively.

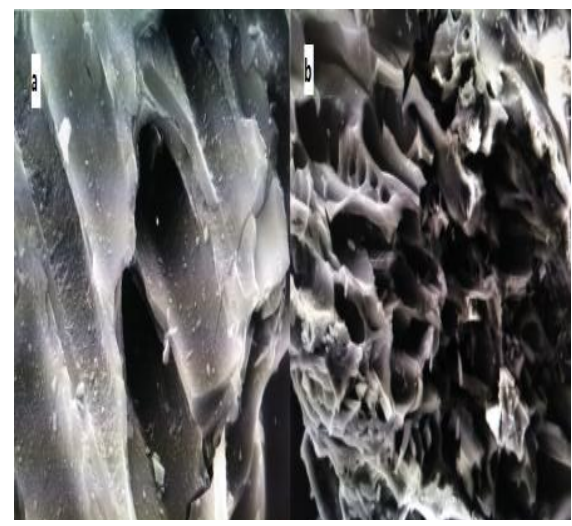

Figure 2 (a)SEM photos before adsorbing acetaldehyde(b)SEM photos after adsorbing acetaldehyde

\section{CONCLUSION}

The CMC/GO-COOH scaffold was prepared by a lyophilization method. The structure of the scaffold exhibited interconnected 3D porous network. The scaffold displayed the best adsorption for formaldehyde. In addition, GO$\mathrm{COOH}$ showed excellent miscibility and dispersion with CMC. The porous structure of the scaffold allowed for the efficient elimination of formaldehyde. In all, the CS/GO$\mathrm{COOH}$ scaffold had good affinity to formaldehyde, and had potential in the application of environment.

\section{ACKNOWLEDGMENTS}

This research was financially supported by the National key research and development program(2016YFC1100704), Beijing municipal science and technology project (Z151100003715006), The capital health research and development of special funding support (2014-1-2091), China Tobacco Guangxi Industrial Co.LTD science and technology project (FZB1401FW017) and Beijing Municipal Administration of Hospictals clinical medicine development of special funding support(ZYLX201508).

\section{REFERENCES}

[1] Mansur S, Othman MHD, Ismail A, et al.Investigation on the effect of spinning conditions on the properties of hollow fiber membrane for hemodialysis application,ApplPolym Sci.133:43633-43634 (2016).

[2] Tetali SD, Jankowski V, Luetzow K, et al. Adsorption capacity of poly (ether imide) microparticles to uremic toxins,Clinical hemorheology and microcirculation 61,1-9 (2015).

[3] Kim K S, Zhao Y, Jang H, et al. Large-scale pattern growth of graphene films for stretchable transparent electrodes,Nature, 457,706-710 (2009).

[4] M.Agarwal, M. Dave and S.Upadhayaya,Adsorption of Formaldehyde on TreatedActivated Carbonand Activated Alumina,Current World Environment6(1), 53-59 (2011). 